\title{
CORRELATION BETWEEN MARGIN FIT AND MICROLEAKAGE IN COMPLETE CROWNS CEMENTED WITH THREE LUTING AGENTS
}

\author{
Paulo Henrique Orlato ROSSETTI ${ }^{1}$, Accacio Lins do VALLE ${ }^{2}$, Ricardo Marins de CARVALHO ${ }^{3}$, \\ Mario Fernando De GOES ${ }^{4}$, Luiz Fernando PEGORARO ${ }^{5}$
}

\begin{abstract}
1- MSc, PhD Student, Oral Rehabilitation Program, Bauru School of Dentistry, University of São Paulo, Bauru, SP, Brazil.
2- MSc, PhD, Professor, Department of Prosthodontics, Bauru School of Dentistry, University of São Paulo, Bauru, SP, Brazil.

3- MSc, PhD, Associated Professor, Department of Prosthodontics, Bauru School of Dentistry, University of São Paulo, Bauru, SP, Brazil.

4- MSc, PhD, Professor, Department of Dental Materials, Piracicaba School of Dentistry, State University of Campinas, Piracicaba, SP, Brazil.

5- MSc, PhD, Titular Professor, Department of Prosthodontics, Dean of the Bauru School of Dentistry, University of São Paulo, Bauru, SP, Brazil.

Corresponding address: Prof. Dr. Luiz Fernando Pegoraro - Faculdade de Odontologia de Bauru - Alameda Dr. Octavio Pinheiro Brisolla,

9-75 - Bauru, São Paulo, Brazil. - e-mail: pegoraro@fob.usp.br
\end{abstract}

Received: July 26, 2007 - Modification: September 18, 2007 - Accepted: October 23, 2007

\begin{abstract}
$M$

icroleakage can be related to margin misfit. Also, traditional microleakage techniques are time-consuming. This study evaluated the existence of correlation between in vitro margin fit and a new microleakage technique for complete crowns cemented with 3 different luting agents. Thirty human premolars were prepared for full-coverage crowns with a convergence angle of 6 degrees, chamfer margin of $1.2 \mathrm{~mm}$ circumferentially, and occlusal reduction of $1.5 \mathrm{~mm}$. Ni-Cr cast crowns were cemented with either zinc phosphate (ZP) (S.S. White), resin-modified glass-ionomer (RMGI) (Rely X Luting Cement) or a resinbased luting agent (RC) (Enforce). Margin fit (seating discrepancy and margin gap) was evaluated according to criteria in the literature under microscope with $0.001 \mathrm{~mm}$ accuracy. After thermal cycling, crowns were longitudinally sectioned and microleakage scores at tooth-cement interface were obtained and recorded at $\times 100$ magnification. Margin fit parameters were compared with the one-way ANOVA test and microleakage scores with Kruskal-Wallis and Dunn's tests $(\alpha=0.05)$. Correlation between margin fit and microleakage was analyzed with the Spearman's test $(\alpha=0.05)$. Seating discrepancy and marginal gap values ranged from $81.82 \mu \mathrm{m}$ to $137.22 \mu \mathrm{m}(\mathrm{p}=0.117)$, and from $75.42 \mu \mathrm{m}$ to $78.49 \mu \mathrm{m}(\mathrm{p}=0.940)$, respectively. Marginal microleakage scores were $\mathrm{ZP}=3.02$, RMGI $=0.35$ and $\mathrm{RC}=0.12$ ( $\mathrm{p}<0.001$ ), with no differences between RMGI and $\mathrm{RC}$ scores. The correlation coefficient values ranged from -0.27 to 0.30 ( $\mathrm{p}>0.05$ ). Conclusion: Margin fit parameters and microleakage showed no strong correlations; cast crowns cemented with RMGI and RC had lower microleakage scores than ZP cement.
\end{abstract}

Uniterms: Dental leakage. Dental cements. Marginal adaptation. Crowns.

\section{INTRODUCTION}

The margin fit of prosthetic crowns has always been a concern for clinicians $2,3,6-9,11,16-18,21,24,30,33,34,37$. Misfit at cementation ${ }^{38}$ results in open margins, increasing the risk of recurrent caries, plaque accumulation and periodontal disease. Several clinical and laboratorial procedures to improve crown seating have been described: chemical or internal relief of casting $\mathrm{s}^{30,34}$, occlusal venting of crowns $^{2,3,16,34}$, the use of a die spacer $^{2,3,9}$, horizontal vibration $^{7,34}$, placement of an internal escape channel in tooth preparation $^{33}$, dynamic seating forces ${ }^{18,24}$, and the use of a silicone disclosing medium ${ }^{38}$ Also, one interesting method integrating silicone material and laser videography has been proposed to determine the precision of fit. ${ }^{20}$ However, the clinical assessment is performed with a sharp probe. Thus, it is impossible to precisely evaluate margin quality after cementation or to eliminate the problem of cement line exposure ${ }^{12,19,22,25,28,29,31,32,35,36,39,41}$.

Microleakage can be related to margin misfit, although no strong correlation between margin fit and microleakage scores in complete crowns has been demonstrated ${ }^{13,19,25,26,30-}$ $32,35,36,39-41$. White et $a l .{ }^{36}$ found that marginal opening did not directly correlate with marginal microleakage. Also, the authors stated that a complex interaction between variables related to dental restoration, luting agent, and tooth structure probably influenced microleakage. Regarding to luting agents, zinc phosphate cement presents higher in vitro 
microleakage scores when compared to ionomeric and resin luting agents. One hypothesis is that the greater solubility of this cement accelerates the chemical dissolution process ${ }^{15}$. However, even with this disadvantage, clinical reports show positive survival rates for complete crowns luted with zinc phosphate cement ${ }^{10,19,29,35,41}$. Adhesive luting agents have been shown to be less soluble, biocompatible, and bacteriostatic $^{27}$. Resin or resin-modified glass ionomer cements have shown lower in vitro microleakage values; however, the long-term in vivo survival rate has not yet been published ${ }^{27}$.

To assess microleakage, crowns are immersed in dye solution for 4 to 72 hours or more ${ }^{1}$. Next, specimens are sectioned and dye penetration is scored along the internal margins. More recently, a new technique described the use of a silver methenamine solution to verify microleakage for adhesive restorations ${ }^{5}$. However, this solution was applied after specimen sectioning and without previous storage. To hypothesize, the use of a dye solution in the same manner could be an easier and faster technique to assess microleakage in complete crowns.

The purpose of this in vitro study was to evaluate the correlation of margin fit and the use of a new technique to assess microleakage after complete crown cementation. The tested null hypotheses were: (1) there is no strong correlation between margin fit and microleakage, and (2) there are no differences in the microleakage values among the materials tested in this technique.

\section{MATERIALAND METHODS}

Thirty human premolars with similar dimensions extracted for orthodontic reasons were used in this study. Each tooth was mounted in a plastic ring (Tigre do Brasil, São Paulo, SP, Brazil; ref. 10120250) with autopolymerized acrylic resin (Classico Artigos Odontológicos Ltda, São Paulo, SP, Brazil), leaving the clinical crown exposed. A condensation silicone index (Zetalabor; Rovigo, Italy; base lot no.19005, catalyst no. 13089) and a scaled periodontal probe (S.S. White, Rio de Janeiro, RJ, Brazil) were used to control tooth reduction. A 1.2-mm diameter diamond bur (no. 3215; KG Sorensen, Barueri, SP, Brazil; lot no.021112) was used to achieve a 6degree converge angle, a chamfer margin of $1.2 \mathrm{~mm}$ circumferentially located at the cementoenamel junction, and an occlusal reduction of $1.5 \mathrm{~mm}$ for each preparation with 4 $\mathrm{mm}$ in height. All line angles were rounded. The first investigator made all preparations and a sharp explorer (S.S. White) was used to detect undercuts at the cervical margins. Preliminary impressions were obtained with irreversible hydrocolloid (Hydrogum, Zermack, Rovigo, Italy; lot. no. A2137B) using metallic stock trays and poured in improved dental stone (Fuji Rock EP; GC Europe, Leuven, Belgium; lot. no.20000511-1). A uniform layer of wax relief was applied to the axial and occlusal walls of each stone die to fabricate 2-mm-thick acrylic custom-made trays. The impressions were made with addition silicone (Express; 3M/ESPE, St Paul, MM, USA; lot no.P001205) material $^{36}$ and dies were fabricated in type IV stone (Vel-mix; Kerr, Romulus, MI, USA, lot no.3-22295). One specialized laboratory technician was involved in waxing, investing and casting all specimens. The patterns were invested with phosphate bondedinvestment (Microfine 1700; Talladium Inc, Valencia, CA, USA; lot no.08093) and cast with a Ni-Cr alloy (Verabond II; AALBA Dent, Cordelia, CA, USA; lot no.040914), according to the manufacturer's recommendations. Crowns were initially adapted to their dies using a uniform layer of liquid disclosing agent (Accufilm IV; Parkell, Farmingdale, NY, USA; stock no.S020) painted on their internal surfaces. After seating, crowns were internally inspected and interferences were removed with a rotary instrument. Then, the internal portions were cleaned with alcohol and the seating procedure was repeated until no detectable interferences were verified. The second and the third investigator controlled casting adaptation. Calibration was done by lightly drawning a sharp explorer back and forth across the cast-tooth interface several times. Final crown adaptation was considered acceptable when no discrepancies were detected. During the entire experiment, specimens remained in distilled water at room temperature.

To evaluate margin fit values, parameters such as seating discrepancy and margin gap were used according to Holmes, et al. ${ }^{14}$. For seating discrepancy measurements, external reference cavities were made with a round no. 2 carbide bur (S.S. White; lot no.020802) at the midpoint of buccal, lingual, mesial and distal surfaces, $1 \mathrm{~mm}$ above and below the toothcrown interface. Afterwards, the cavities were filled with autopolymerized acrylic resin (Duralay; Reliance Dental Mfg Co, Worth, IL, USA; reorder no.2244). All recordings were made under a measuring microscope with $0.001 \mathrm{~mm}$ accuracy (Model 505, Mitutoyo, Tokyo, Japan; code 176-811A), using the inferior and superior tangents to the acrylic filled upper and lower cavities. The tangent lines were obtained with the cross-hair reticule of the microscope. A special plastic jig was made to assure measurement accuracy at the desired points before and after crown cementation: the PVC rings were attached perpendicular to the arm of a paralellometer. Second, the lateral side of an acrylic rectangular box was trimmed to match the PVC ring. After, the box was filled with addition silicone material. Then, the PVC ring was immersed into the box with silicone material, creating a negative impression. In this way, all PVC rings were allowed to be positioned at the same place during all measurements. The vertical distance perpendicular to the superior and inferior tangents corresponded to the seating discrepancy measurements (Figure 1). Three measurements were made between these 2 reference lines on each designated surface of the tooth, by the second and the third investigator. Thereafter, the specimens were distributed into 3 groups: zinc phosphate (ZP) (S.S. White; lot no. 8342211), resinmodified glass-ionomer (RMGI) (Rely X Vitremer Luting Cement; 3M/ESPE; lot no. 20000821) and a resin luting agent (RC) (Enforce; Dentsply, Petropolis, RJ, Brazil; lot no.67018). After mixing the cement following the manufacturer's recommendations, a thin layer of cement was painted on the internal surfaces of the crowns. Crowns were initially seated 
on the prepared teeth with finger pressure and sustained with a $5 \mathrm{~kg}$ load for 10 minutes $^{23}$. Buccal, lingual, mesial and distal tooth-crown margins were photopolymerized at 1-mm distance for 40 seconds each (Optilux; Dabi Atlante, Ribeirao Preto, SP, Brazil) with a light intensity of $400 \mathrm{~mW} / \mathrm{cm}^{2}$. After 24 hours, the seating discrepancy measurements were repeated again three times as already described. The difference between pre- and post-cementation collated values was defined as the definitive seating discrepancy values. The first investigator made all measurements. Only buccal and lingual values were considered for analysis. Specimens were subjected to 300 thermal cycles between $5^{\circ} \mathrm{C}$ and $55^{\circ} \mathrm{C}$ with a 30 second dwell time and stored in distilled water for 24 hours at room temperature.

Margin gap assessment was performed after sectioning the specimens in a midbucco-lingual plane with a low-speed diamond saw (Extec Diamond Wafering Blade; Extec Co, Enfield, CT, USA; ref. no.12205) parallel to the long axis of the cast. Margin gap was considered as the perpendicular measurement from the internal surface of the casting to the margin of the preparation ${ }^{14}$. The point of closest

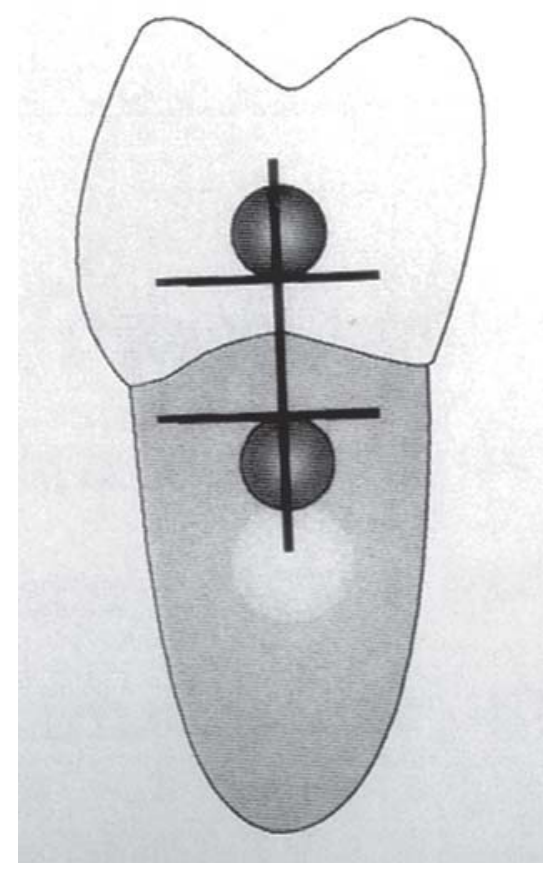

FIGURE 1- External reference marks from a distal view on crown and tooth $1.0 \mathrm{~mm}$ from the margin to quantify seating discrepancy measurements on all four surfaces of tooth approximation between the casting and margin was selected ${ }^{20}$. Each measurement was repeated three times for buccal and lingual margins. Margin gap values were recorded under a digital microscope camera at $\times 100$ magnification (Microscope BeautyCam; Ribeirão Preto, SSP, Brazil). Specimens were rinsed in distilled water and ultrasonically cleaned. To evaluate margin microleakage, a dye composed of red 52 (1\% Rhodamine B) acid solution (Caries Detector; Kuraray Co, Okayama, Japan; lot no.0530D) and propylene glycol was applied for 15 seconds to the intaglio portion of crown along the tooth-cement interface, and washed with distilled water for 15 seconds. The presence of microleakage was confirmed by the visualization of a purple colored reaction at the tooth-cement interface. The first and the fourth investigators were involved in microleakage evaluation. Microleakage patterns were fully registered on the buccal and lingual margins with the same digital microscope camera at $\times 100$ magnification. Microleakage was scored using Tjan's et al. $\operatorname{method}^{31}$ : $0=$ no microleakage; $1=$ microleakage to one-third of axial wall; $2=$ microleakage to two thirds of axial wall; $3=$ microleakage along the full length of axial wall, and $4=$ microleakage over the occlusal surface.

All data analyses were made with SigmaStat 2.0 statistical software (Jandel Corporation, CA, USA). Mean and standard deviations for each marginal fit parameter and microleakage scores were calculated. Statistical analysis for margin fit parameters was performed by one-way ANOVA. KruskalWallis and Dunn's tests were used to identify statistically significant differences in microleakage scores. Spearman's rank test was used to assess the existence of correlation between margin fit parameters and microleakage. Significance level was set at $\mathrm{a}=0.05$ for all statistical tests.

\section{RESULTS}

Means and SD for margin fit parameters and microleakage scores are shown in Table 1. The seating discrepancy values ranged from 81.82 to $137.22 \mu \mathrm{m}(\mathrm{p}=0.117)$, while the margin gap values ranged from 75.42 to $78.49 \mu \mathrm{m}$ ( $p=0.940)$ for the three cements. RC cement displayed the smallest mean seating discrepancy and margin gap values. The mean margin microleakage scores were $\mathrm{ZP}=3.02$, RMGI $=0.35$ and $\mathrm{RC}=0.12$ (p<.0001) (Table 1). Only RMGI and RC scores had no statistically significant differences to each other. The correlation coefficient values ranged from 0.27 to 0.30 ( $>>0.05)$ (Table 2). In some cases, the strength of

TABLE 1- Means and SD of seating discrepancy, marginal gap (in $\mu \mathrm{m}$ ) and microleakage scores

\begin{tabular}{lccc} 
Groups & Seating discrepancy & Marginal gap & Microleakage \\
\hline ZP & $142.50 \pm 102.33$ & $78.39 \pm 24.54$ & $3.02 \pm 0.89 \mathrm{a}$ \\
RMGI & $111.16 \pm 88.54$ & $78.49 \pm 33.53$ & $0.35 \pm 0.48 \mathrm{~b}$ \\
RC & $84.90 \pm 64.05$ & $75.42 \pm 35.19$ & $0.12 \pm 0.27 \mathrm{~b}$ \\
\hline
\end{tabular}

Different letters in the same column indicate statistically significant differences (Kruskal-Wallis, $\mathrm{p}<.001$ ). 
TABLE 2- Correlation values between margin fit parameters and microleakage

Seating discrepancy and microleakage

\begin{tabular}{lllll}
\hline Groups & $r$ & $p$ & $r$ & $p$ \\
ZP & -0.01 & 0.95 & -0.27 & 0.22 \\
RMGI & 0.30 & 0.19 & 0.26 & 0.25 \\
RC & 0.13 & 0.57 & 0.07 & 0.74 \\
\hline
\end{tabular}

association between margin fit and microleakage is weak and negative, with one always decreasing as the other increases. In other cases, this strength is weak and positive, with both always increasing together.

\section{DISCUSSION}

Measurement techniques and the descriptive terminology of fit for complete crowns varies among the investigators $2,3,6-9,11,16-18,21,24,30,33,34$. Criteria proposed by Holmes, et al. ${ }^{14}$ are useful for determining the seating discrepancy and margin gap values. In this study, both margin fit values of the tested cements were similar to those reported on previous investigatons $s^{6,8,9,20,21,24-27,34,36-38}$.

The large variation observed for seating discrepancy ( 71.6 to $82.8 \%$ ) and margin fit ( 32.3 to $48.4 \%$ ) for the 3 tested cements is related to the oblique seating of crowns during cementation $^{38}$. This re-emphasizes that margin fit values show to what extent the crown is obliquely seated, but they do not reflect the distribution of the cement layer around the restoration. Perhaps this variation was due to the fewer measurements for both margin fit values ${ }^{12}$.

One important aspect that deserves attention is the lack of significant differences for intergroup comparisons of margin fit values. Factors such as preparation type, internal adjustment of crowns during seating procedure, cementation load and time would not be enough to generate similar viscosities after manipulation of these cements. Furthermore, the number of layers of die relief spacer was the same for the 3 groups.

To verify the margin microleakage, a dye was applied directly to the internal portion of crown at the tooth-cement interface. The advantage of this technique is that dye penetration can be seen more rapidly and in a shorter exposure time (15 seconds). However, it was not the silver methenamine used in the literature ${ }^{5}$. Nevertheless, the manufacturer purports that Caries Detector is a solution and has no filler, so no particle size. Thus, a shorter exposure time is enough to visualize it.

Fick's first law of diffusion ${ }^{27}$ states that the rate of material dissolution is independent of the exposed area (amount of luting agent). Correlation values between misfit and microleakage were low because the gap formation at the tooth-cement interface partially accounts for the microleakage values observed. This is supported by the presence of largest mean seating discrepancy occurring with the ZP cement. The bond quality of the cement could have a significant role in the microleakage as well, since the test cements were resin based cements.

This in vitro study showed that there is no strong correlation between margin fit parameters and microleakage in this technique. Thus, the first hypothesis is accepted. This observation confirms the findings of White, et al. ${ }^{36}$ and more recently, the study conducted by Piwowarczyk, et al. ${ }^{26}$ However, the problem of marginal gap and leakage has challenged the profession for decades. First, currently available cements are soluble and do not provide continual impermeability at tooth-cement interface. In addition, open margins increase the risk for caries, cervical discoloration, pulp necrosis, and loss of restoration. Additionally, techniques to improve crown seating just minimize this problem. Therefore, clinicians must expect some degree of tilting and should strive to reduce margin misfit. This problem may be aggravated in fixed partial restorations with vital abutments. Nevertheless, clinicians should strive to minimize margin misfit. Cement excesses are difficult to remove and create potential areas for plaque accumulation.

There are limitations to this study. First, there is concern about cement layer integrity after crown sectioning. Even with low-speed sectioning it is possible for disc to remove a portion of the cement layer. However, microleakage assessment is performed adjacent to the sectioned surface. A second concern is that the distilled water involved in the cutting process could affect water-based cements. Resinmodified glass ionomer cements are sensitive to moisture, resulting in crack formation ${ }^{27}$. To avoid this, all exposed areas were stored in distilled water. Before microleakage assessment, care was taken to gently remove water excess. Visualization with the optical microscope revealed no cracks. Furthermore, for the RC group, it may not have been possible to properly polymerize the cement bulk beyond the margins of a complete crown. During storage, the water sorption process could cause expansion of the cement layer and close the gaps at tooth-cement interface. Even with these limitations, margin microleakage values were similar to that described in the literature ${ }^{13,31,32,36,39}$.

The zinc phosphate cement showed higher margin microleakage scores in relation to RMGI and RC cements. Thus, the second part of the hypothesis has to be rejected. However, clinical success of ZP cements does not correlate to the laboratory findings. Still, they are easy to manipulate and do not involve acid etching procedures that are operator sensitive. By contrast, microleakage values for RMGI and 
$\mathrm{RC}$ cements were lower. This may have been due to their lower solubility and higher water sorption, which would have closed the gaps by expansion of the cement layer. In addition, ability to adhere to tooth substrate with dental conditioning is relevant. Thus, lack of retention is minimized. However, this study verified short-term responses of adhesive interfaces to microleakage. Water storage for longer time periods could provide higher microleakage values. Newer adhesive systems available today do not have clinical studies to support their effectiveness. Thus, more clinical trials are necessary in full crown adhesive cementations. Higher standard deviations in the margin microleakage scores were seen for the RMGI and RC cements (Table 1). However, fewer specimens in the RMGI (3 of 10) and RC (5 of 10) groups presented microleakage. In the ZP group, only one specimen showed no microleakage.

Dye penetration at tooth margins would be potentially less harmful to the pulp than the rest of the preparation, due to the small number of dentinal tubules present in this area. However, dye at the tooth-cement interface indicates that potential gaps are present and they may harbor bacteria that would eventually cause caries and periodontal disease ${ }^{13}$. Although there is a complex relationship among diet, substrate, and time, gingival health largely depends on hygiene procedures. This fact is aggravated because direct visualization is not possible in subgingival crown margins. In addition, non-polymerized resin components into the gingival sulcus could cause inflammatory reaction ${ }^{23}$, color changes $^{4}$ and loss of the restoration. Moreover, most specimens in the ZP group showed extended microleakage into the dentinal tubules. This rarely was observed in RMGI and $\mathrm{RC}$ cements. In the worst scenario, the inability of ZP to seal the dentinal tubules may suggest potential harm to the pulp.

The use of this technique requires careful observation of the stained area: dye application must be continuous, beginning at the margins, traveling from one side to another. Dye solution will stain porosities in the bulk of cement layer, and gap formation at tooth-cement interface. This is explained because $1 \%$ Rhodamine B is water-soluble and reacts chemically with $\mathrm{ZP}$ and RMGI producing a purple color appearance. For the ZP group, the entire cement layer was stained, but only the tooth-cement interface must be considered. For the RMGI specimens, the staining of the material was less intense. Most specimens showed no staining. In the RC group, dye concentrated only at the tooth-cement interface and hardly on cement porosities. These observations are necessary to interpret margin microleakage and exclude false results.

\section{CONCLUSIONS}

Within the limits of this study, the following conclusions can be drawn: 1 . No strong correlation was found between margin fit parameters and microleakage; 2. Cast crowns cemented with RMGI and RC demonstrated significantly lower microleakage those cemented with ZP cement.

\section{REFERENCES}

1- Alani AH, Toh CG. Detection of microleakage around dental restorations: a review. Oper Dent. 1997;22:173-85.

2- Bassett RW. Solving the problems of cementing the full veneer cast gold crown. J Prosthet Dent. 1966;16:740-7.

3- Cooper TM, Christensen GJ, Laswell HR, Baxter R. Effect of venting on cast gold full crowns. J Prosthet Dent. 1971;26:621-6.

4- Darr AH, Jacobsen PH. Conversion of dual cure luting cements. J Oral Rehabil. 1995;22:43-47.

5- De Góes MF, Montes MAJR. Evaluation of a silver methenamine method for nanoleakage. J Dent. 2004;32:391-8.

6- Dimashkieh MR, Davies EH, von Fraunhofer JA. Measurement of the cement film thickness beneath full crown restorations. Br Dent J. 1974;137:281-4.

7- Eames WB, O’Neal SJ, Monteiro J, Miller C, Roan Jr JD, Cohen KS. Techniques to improve the seating of castings. J Am Dent Assoc. 1978;96:432-7.

8- Fusayama T, Ide K, Hosoda H. Relief of resistance of cement of full cast crowns. J Prosthet Dent. 1964;14:95-106.

9- Gegauff AG, Rosenstiel, SF. Reassessment of die-spacer with dynamic loading during cementation. J Prosthet Dent. 1989;61:655-8.

10- Goodacre CJ, Bernal G, Rungcharassaeng K, Kan JYK. Clinical complications in fixed prosthodontics. J Prosthet Dent. 2003;90:3141 .

11- Grajower R, Lewinstein I, Zeltser C. The effective minimum cement thickness of zinc phosphate cement for luted non-precious crowns. J Oral Rehabil. 1985;12:235-45.

12- Groten M, Axmann D, Pröbster L, Weber H. Determination of the minimum number of marginal gap measurements required for practical in vitro testing. J Prosthet Dent. 2000;83:40-9.

13- Gu X, Kern M. Marginal discrepancies and leakage of all-ceramic crowns: influence of luting agents and aging conditions. Int $\mathrm{J}$ Prosthodont. 2003;16:109-116.

14- Holmes JR, Bayne SC, Holland GA, Sulik WD. Considerations in measurement of marginal fit. J Prosthet. Dent. 1989;62:405-8.

15- Jacobs MS, Windeler AS. An investigation of dental luting cement solubility as a function of the marginal gap. J Prosthet Dent. 1991;65:436-42.

16- Jones MD, Dykema RW, Kein AI. Television micromeasurement of vented and non-vented cast crown marginal adaptation. Dent Clin North Am. 1971;15:663-78.

17-Jorgensen KD. Factors affecting the film thickness of zinc phosphate cements. Acta Odontol Scand. 1960;18:479-90.

18- Kaufman EG, Coelho DH, Colin L. Factors influencing the retention of cemented gold castings. J Prosthet Dent. 1961;11:487502 .

19- Kidd WL, Nicholls JI, Harrington G, Freeman M. Marginal leakage of cast gold crowns luted with zinc phosphate cement: an in vivo study. J Prosthet Dent. 1996;75:9-13.

20- May KB, Russell MM, Razzoog ME, Lang BR. Precision of fit: the Procera Allceram crown. J Prosthet Dent. 1998;80:394-404. 
21 - McLean JW, von Fraunhofer JA. The estimation of cement film thickness by an in vivo technique. Br Dent J. 1971;131:107-11.

22 - Mitchell CA, Pintado MR, Douglas WH. Nondestructive, in vitro quantification of crown margins. J Prosthet Dent. 2001;85:575-84

23- Peumans M, Van Meerbeek B, Lambrechts P, Vanherle G, Quirynen $\mathrm{M}$. The influence of direct composite additions for the correction of tooth form and/or position on periodontal health. A retrospective study. J Periodontol. 1998;69:422-7.

24- Pilo R, Cardash HS, Baharav H, Helft M. Incomplete seating of cemented crowns: a literature review. J Prosthet Dent. 1988;59:42933 .

25 - Pilo R, Cardash HS. In vivo retrospective study of cement thickness under crowns. J Prosthet Dent. 1998;79:621-5.

26- Piwowarczyk A, Lauer HC, Sorensen JA. Microleakage of various cementing agents for full cast crowns. Dent Mat. 2005;21:445-53.

27- Rosenstiel SF, Land MF, Crispin BJ. Dental luting agents: a review of the current literature. J Prosthet Dent. 1998;80:280-301.

28 - Roulet JF. Marginal integrity: clinical significance. J Dent. 1994;22:9-12.

29- Schwartz NL, Whitsett LD, Berry TG, Stewart JL. Unserviceable crowns and fixed partial dentures: life-span and causes for loss of serviceability. J Am Dent Assoc. 1970;81:1395-401.

30-Selberg A. A full cast crown technique. J Prosthet Dent 1957;7:102-22.

31- Tjan AHL, Dunn JR, Grant BE. Marginal leakage of cast gold crowns luted with an adhesive resin cement. J Prosthet Dent. $1992 ; 67: 11-5$

32- Tjan AHL, Peach KD, VanDenburgh SL, Zbaraschuk ER Microleakage of crowns cemented with glass ionomer cement: effects of preparation finish and conditioning with polyacrylic acid. J Prosthet Dent. 1991;66:602-6.

33- Tjan AHL, Sarkissian R. Internal escape channel: an alternative to venting complete crowns. J Prosthet Dent. 1984;52:50-6.

34- Van Nortwick WT, Gettleman L. Effect of internal relief, vibration, and venting on the vertical seating of cemented crowns. J Prosthet Dent. 1984;45:395-9.

35- Walton JN, Gardner FM, Agar JR. A survey of crown and fixed partial denture failures: length of service and reasons for replacement. J Prosthet Dent. 1986;56:416-21.

36- White SN, Ingles S, Kipnis V. Influence of marginal opening on microleakage of cemented artificial crowns. J Prosthet Dent. 1994;71:257-64.

37- White SN, Kipnis V. Effect of adhesive luting agents on the marginal seating of cast restorations. J Prosthet Dent. 1993;69:2831 .

38- White SN, Kipnis V. The three-dimensional effects of adjustmen and cementation on crown seating. Int J Prosthodont. 1993;6:24854.

39- White SN, Sorensen JA, Kang SK, Caputo AA. Microleakage of new crown and fixed partial denture luting agents. J Prosthet Dent. 1992;67:156-61.
40- White SN, Yu Z, Tom JF, Sangsurasak S. In vivo marginal adaptation of cast crowns luted with different cements. J Prosthet Dent. 1995;74:25-32.

41 - White SN, Yu Z, Tom JF, Sangsurasak S. In vivo microleakage of luting cements for cast crowns. J Prosthet Dent. 1994;71:333-8. 